\title{
Fourier analysis of polar coordinate data in visual physiology and psychophysics
}

\author{
ROBERT SEKULER AND ROBERT ARMSTRONG \\ Northwestern University, Evanston, Illinois 60201
}

\begin{abstract}
Techniques are presented for analyzing data collected as a function of: a circular independent variable (e.g., angle, direction, or orientation) or an independent variable which is cyclical (e.g., months of the year). The procedures are presented in a tutorial manner, emphasizing their relationship to more traditional statistics in experimental psychology.
\end{abstract}

Studies of visual physiology or psychophysics frequently employ dependent measures which are some function of angle or direction of motion. In this paper we shall outline a simple statistical approach to the special problems raised by such data. No claims are made for the uniqueness of the approach (Mardia, 1972); we seek only to present the procedure in as accessible a manner as we can, given the limitations of space.

Consider some examples of the class of studies we address. Barlow, Hill, and Levick (1964) measured the responses of rabbit retinal ganglion cells to spots of luminance which moved in various directions. In an analogous human psychophysical study, Levinson and Sekuler (Note 1) measured contrast detection thresholds for dots moving in various directions, before and after direction-specific adaptation. Appelle (1972) summarized a large number of experiments designed to measure sensitivity to grating and line stimuli presented in various orientations. In vision clinics, perimetric techniques are often used to define various isopters, contours along which visual sensitivity is constant; the shape of the isopters may be diagnostically significant (Frisen \& Frisen, 1975). Finally, although it does not use an independent variable which is a function of angle, the Farnsworth-Munsell test of hue discrimination produces data which are usually presented as a function of angle, on circular coordinates (Adams, Brown, Haegerstrom-Portnoy, Flom, \& Jones, 1976). ${ }^{1}$

In all these and related studies, the investigators wanted to know: (1) whether the various orientations or directions produce reliably different responses and, if they did, (2) how best to characterize the pattern of responses as a function of angle. Of course such a characterization can have theoretical as well as descriptive importance. In the case of visual cortical

We thank Ms. Frances Levitt, Dr. Robert Emerson, and Dr. John Lott Brown for allowing us to analyze and describe some of their excellent data. We also thank Dr. John Tuttle of the University of Rochester for checking the accuracy of our calcu. lations and optimizing our computer program. Preparation of this paper was supported by NSF Grant BNS77-15858 and NIH Grant EY-00321. neurons, this characterization might describe a cell's axis (orientation) preference, its directional bias, and the narrowness of each.

The experimental situations we are considering have two peculiarities which make standard well-known statistical approaches inappropriate. First, the data are collected on a circle. As a consequence, functions chosen to represent the data must be periodic. Obviously the functions must have the same value at $\theta$ and at $\theta+2 \pi$. This restriction rules out the use of traditional decompositions as orthogonal polynomials. Although polynomial trend vectors could $\operatorname{span}^{2}$ the space of data patterns, they would do so in a highly artificial way since odd terms would generate patterns with large discontinuities at $\theta=2 \pi$. Second, since the experiments were designed and the data collected with a circular representation in mind, an analysis which preserves the polar coordinate character of the data seems more natural. In face, a circular, polar representation of ten reveals features of the data that would be obscured on rectilinear coordinates. This fact, too, mandates the use of a basis ${ }^{3}$ set which is natural to polar coordinates.

Procedures for analyzing data on polar coordinates are described best in the context of particular results. We shall begin by considering some experiments of Levitt, Emerson, and Brown at the University of Rochester (Note 2). Levitt and her colleagues stimulated cats' eyes with a moving square-wave grating $(.5 \mathrm{cycle} / \mathrm{deg})$ and recorded responses thereto from single cortical cells. The grating's orientation and direction of movement were controlled by a laboratory computer. At each of 128 equally spaced, different orientations, the grating moved first in one direction normal to its orientation, then in the opposite. The cell's spike responses were partitioned according to the direction of movement which had evoked them. Figure 1 shows spike frequency histograms for four cells plotted on circular or polar coordinates. Data points are evenly spaced around the polar system, at intervals of $2 \pi / 256$ radians. Throughout most of the remainder of this paper, the data shown in Figure 1 will be analyzed and described.

We shall apply the general linear model of analysis 


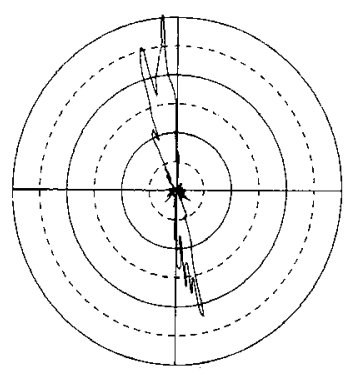

CELL $57-2-1$

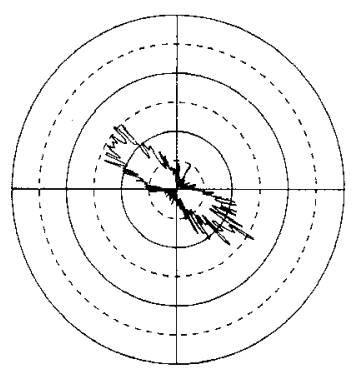

CELL 73-1-1

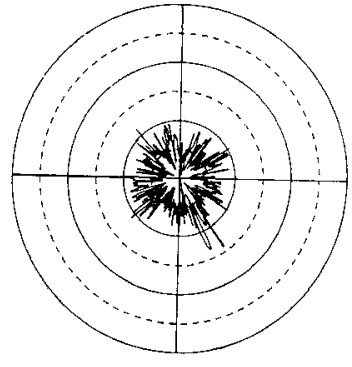

CELL 88-7-1

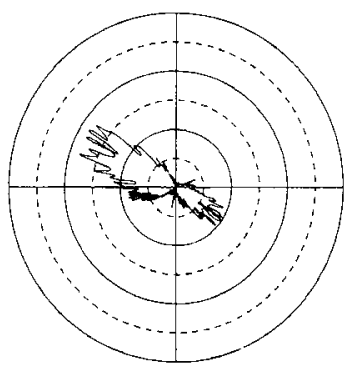

CELL 58-3-1
Figure 1. Polar plots showing response frequency histograms for four cat cortical cells. Data vectors for all cells were normalized to the same length; as a result, differences among cells' mean firing rates are removed. See text for further details. Data courtesy of Drs. Levitt, Emerson, and Citron.

of variance (Dwass, 1970; Scheffé, 1959) to polar data. As in other procedures for partitioning experimental variance into orthogonal components, a set of basis vectors must be chosen to span the space containing all data patterns which one would consider likely a priori, assuming error-free data. A subset of these vectors spans the space of data patterns which defines the null hypothesis, $\mathrm{H}_{0}$ : the remaining vectors of the set define the alternate hypothesis, $\mathrm{H}_{1}$. Each of the $\mathrm{H}_{1}$ vectors must be orthogonal to each of the $\mathrm{H}_{0}$ vectors, and vice versa. ${ }^{4}$

In the Levitt et al. (Note 2) experiment, $\mathrm{H}_{0}$ would hold that the direction of movement had no effect on a cell's response. Formally, if $\mathrm{d}$ is the 256-element vector of obtained data ordered by angle $\theta$, where $\theta=0,2 \pi / 256, \ldots,(2 \pi \cdot k) / 256, \ldots,(2 \pi \cdot 255) / 256$, $\mathrm{H}_{0}$ asserts that $\mathrm{d}=\mathrm{c}+\mathrm{e}$, where $\mathrm{c}$ is a constant vector which, in polar coordinates, plots as a figure (circle) of constant radius and $\mathrm{e}$ is a vector of random deviations from that circle. We also make the standard assumptions that the deviations, $e_{i}, i=0,1, \ldots, 255$, are independent, identically distributed Gaussian variables with $\mu=0$.

More generally, taking both $\mathrm{H}_{0}$ and $\mathrm{H}_{1}$ into account, the linear model assumes $d=A \beta+e$, where $d$ and e are as before and $A \beta$ is the vector of expected values for the components of $\mathrm{d}$. $\mathrm{A}$ is the matrix whose columns are the vectors which span the space of patterns, allowed under $\mathrm{H}_{0}$ and $\mathrm{H}_{1} . \beta$ contains the coefficients which determine each column's contribution to the pattern (ignoring error, e).

From the infinite number of possible basis sets, we chose to work with the terms of a Fourier series:

$$
\mathrm{d}_{\mathrm{i}}(\theta)=\mathrm{c}+\sum_{\mathrm{i}=\mathrm{i}}^{\mathrm{n}}\left(\mathrm{a}_{\mathrm{i}} \cos \mathrm{i} \frac{\theta}{2 \pi}+\mathrm{b}_{\mathrm{i}} \sin \mathrm{i} \frac{\theta}{2 \pi}\right) \text {. }
$$

As shall become apparent, sine and cosine terms ${ }^{5}$ of various frequencies provide a natural basis for the patterns of data most likely to occur in psychophysics and sensory physiology. For the present, note that the mean term, $c$, of the Fourier series represents the null hypothesis mentioned earlier. Note also that sine and cosine terms progress from representing rather global deviations from circularity (low-frequency terms) to reflecting increasingly local deviations from circularity (high-frequency terms).

Figures $2 a-2 d$ are polar coordinate plots of sine functions of period $2 \pi$ through $\pi / 2$; Figures $3 a-3 d$ are corresponding plots for cosine functions (in each case, a circle of unit radius has been added to the functions to avoid negative values). These functions can be considered the canonical shapes for our analysis; we will be regressing data on each one of these functions. A nonzero regression of the data on any of the sine or cosine functions reflects the presence and amplitude of that function in the data. It should be possible, as in
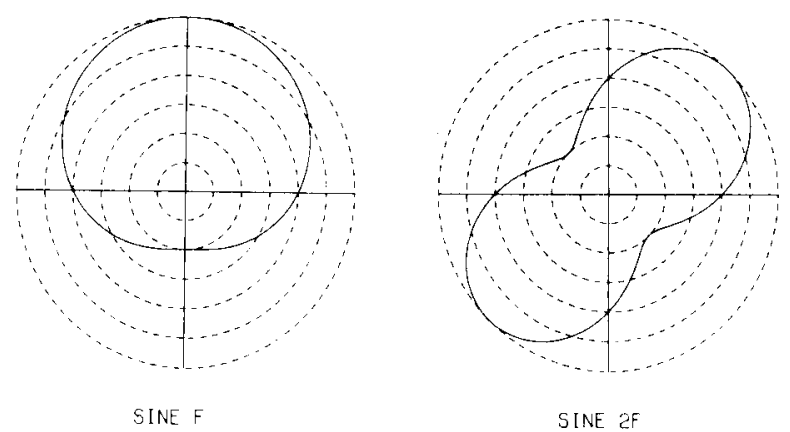

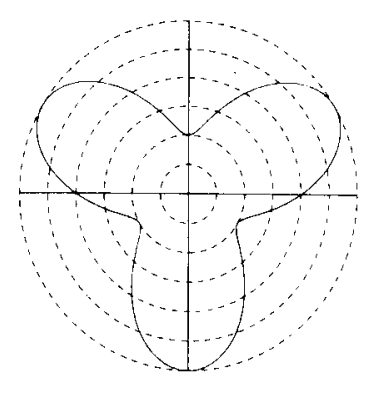

SINE 3F

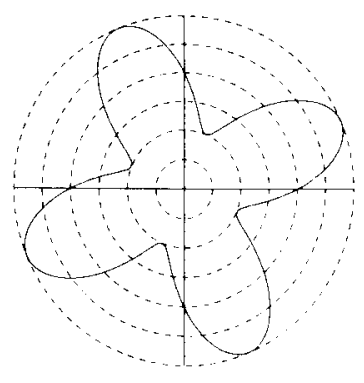

SINE UF
Figure 2. Polar plots of sine functions of frequency $F, 1 F$, $3 F$, and 4F. In each case, a constant (1.0) has been added to avoid negative values; reference circles are drawn at intervals of .5 . 


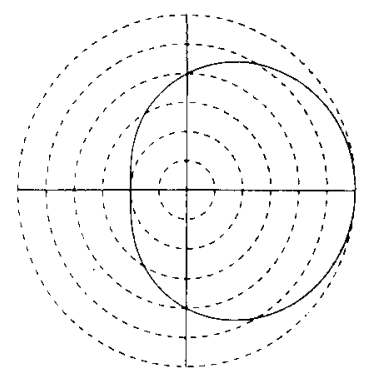

COSINE F

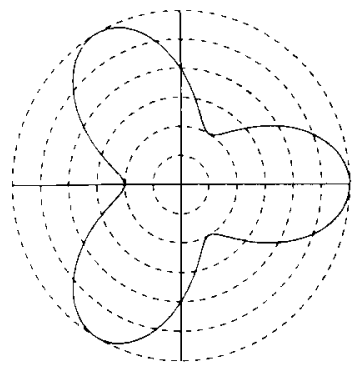

COSINE $3 F$

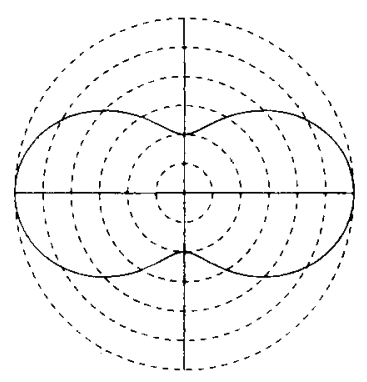

COS:NE $2 F$

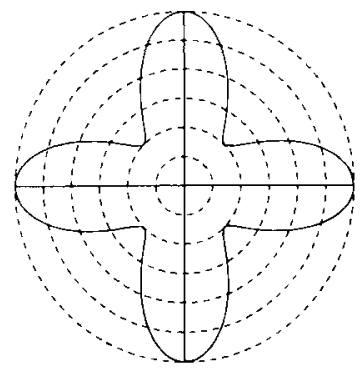

COSINE $4 F$
Figure 3. Same as Figure 2 but for cosine functions.

other types of Fourier analysis, to describe any set of data as the sum of a circle term plus varying amplitudies of shapes like those shown in Figures 2 and 3. A detailed interpretation of each shape's meaning will be given later. First, let us consider one strategy for Fourier analyzing the data of Figure 1.

\section{CALCULATION PROCEDURE}

To estimate the magnitude of various Fourier components in the data, we calculated the Pearson productmoment correlation between a cell's responses and that Fourier component. ${ }^{6}$ The numerical technique, chosen for expositional clarity rather than computational efficiency, required the construction of two vectors: $d$, a vector of data (the cell's responses), and $t$, a vector containing the Fourier term of interest. We normalized $d$ to a vector of unit length, $d^{\prime}$, by computing $d^{\prime}=d /\|d\|$ where $\|d\| l$ is the length of $d$ and is defined to be the square root of the sum of the squares of the elements of $d$. In our case,

$$
\|d\|=\sum_{\mathrm{i}=1}^{256} \mathrm{~d}_{\mathrm{i}}^{2} .
$$

So, the $\mathrm{i}^{\text {th }}$ element of $\mathrm{d}$ is

$$
d_{i}^{\prime}=\frac{d_{i}}{\left[\sum_{j=1}^{256} d_{j}^{2}\right]^{0.5}}
$$

In the same way, $\mathrm{t}$ was normalized to a vector of unit length, $t^{\prime}$. The Pearson product-moment correlation was then obtained by computing the inner product of $t^{\prime}$ and $\mathrm{d}^{\prime}$,

$$
r=\sum_{i=1}^{256} d_{i}^{\prime} t_{i}^{\prime}
$$

A correlation obtained with $t=\cos i \frac{\theta}{2 \pi}$ gives the $a_{i}$ weight for the Fourier series; a correlation obtained with $t=\sin i \frac{\theta}{2 \pi}$ gives a $b_{i}$ weight for the series. To complete the Fourier series, we computed $c$, the circle term. A vector composed of all ones was normalized to unit length (which made each element $=1 / \sqrt{256}$ ). The inner product of this normalized vector and $t$ ' gives the circle term directly.

Table 1 provides the product-moment correlations, r, for Cell 58-3-1; Table 2 does the same for Cell 88-7-1. Recall that these correlations constitute $c, a_{n}$ and $b_{n}$ for the cell's Fourier series (Equation 1). To show what proportion of a cell's total variance each Fourier term accounts for, we have also computed $\mathrm{r}^{2}$. For example, Table 2 shows that the circular term accounts for nearly $86 \%$ of Cell $88-7-1$ 's variance.

\section{INTERPRETATION OF THE FUNDAMENTAL TERMS}

To see concretely what aspect of the data structure each series term reflects, consult Figures 2 and 3 . The fundamental components (sine $F$ and $\cos F$ ) of the Fourier series measure the disparity between the centroid of the data and the origin of the coordinate system. If neither fundamental term was significantly different from zero, the middle of the data would not be significantly removed from the origin. In Levitt et al.'s (Note 2) data this would signify an absence of directional selectivity: Responses produced by movement within any one semicircle would have been balanced by responses to movement within the opposite semicircle.

The relative magnitudes of the fundamental terms

Table 1

Cell 58-3-1: Pearson Product-Moment Correlations (r) and Percent Variance $\left(\mathrm{r}^{2}\right)$ Accounted for by Various Terms of the Fourier Series

\begin{tabular}{lrc}
\hline \multicolumn{1}{c}{ Series Term } & $\mathrm{r}$ & \multicolumn{1}{c}{$\mathrm{r}^{2}$} \\
\hline Circle $(\mathrm{c})$ & .6718 & .4513 \\
Cosine $\mathrm{F}\left(\mathrm{a}_{1}\right)$ & -.2824 & .0798 \\
Cosine $2 \mathrm{~F}\left(\mathrm{a}_{2}\right)$ & .3925 & .1540 \\
Cosine $3 \mathrm{~F}\left(\mathrm{a}_{3}\right)$ & -.1100 & .0121 \\
Cosine $4 \mathrm{~F}\left(\mathrm{a}_{4}\right)$ & -.0617 & .0038 \\
Sine $\mathrm{F}\left(\mathrm{b}_{2}\right)$ & .0336 & .0011 \\
Sine $2 \mathrm{~F}\left(\mathrm{~b}_{2}\right)$ & -.4392 & .1929 \\
Sine $3 \mathrm{~F}\left(\mathrm{~b}_{3}\right)$ & .1158 & .0134 \\
Sine $4 \mathrm{~F}\left(\mathrm{~b}_{4}\right)$ & -.2075 & .0430 \\
Sum & & .9514 \\
\hline
\end{tabular}


Table 2

Cell 88-7-1: Pearson Product-Moment Correlations (r) and Percent Variance $\left(r^{2}\right)$ Accounted for by Various Terms of the Fourier Series

\begin{tabular}{lrc}
\hline Series Term & \multicolumn{1}{c}{$r$} & $\mathbf{r}^{2}$ \\
\hline Circle $(\mathrm{c})$ & .9258 & .8572 \\
Cosine $\mathrm{F}\left(\mathrm{a}_{1}\right)$ & -.0024 & .0000 \\
Cosine 2F $\left(\mathrm{a}_{2}\right)$ & .0144 & .0002 \\
Cosine $3 \mathrm{~F}\left(\mathrm{a}_{3}\right)$ & -.0022 & .0000 \\
Cosine 4F $\left(\mathrm{a}_{4}\right)$ & -.0350 & .0012 \\
Sine F $\left(\mathrm{b}_{1}\right)$ & .0027 & .0000 \\
Sine 2F $\left(\mathrm{b}_{2}\right)$ & -.0344 & .0012 \\
Sine 3F $\left(\mathrm{b}_{3}\right)$ & -.0159 & .0003 \\
Sine 4F $\left(\mathrm{b}_{4}\right)$ & .0705 & .0050 \\
Sum & & .8650 \\
\hline
\end{tabular}

indicate the direction of the centroid's departure from the origin. For example, if the sine $F$ term were greater than zero but the $\cos F$ term were zero, the data centroid would lie along the line from the origin to $(\mathrm{x}=0$, $y>0$ ). If both sine $F$ and $\cos F$ were nonzero, their ratio would be the tangent of the direction in which the centroid went out from the origin and the quadrant within which the direction lies could be determined from the signs of the sine and cosine fundamentals.

Figure 4 shows how several different combinations of sine $F$ and $\cos F$ terms would produce data which depart from the origin in different directions. In fact, any direction could be produced by an appropriately chosen combination. To be more concrete, consider Cell 58-3-1 (Table 1). Its sine $F$ term $=.0336$; its $\cos \mathrm{F}$ term $=-.2824$. The inverse tangent of $(.0336 /-.2824=-6.82 \mathrm{deg}$. A positive sine and a negative cosine places the angle in quadrant II; the direction of displacement is therefore $6.82 \mathrm{deg}$ clockwise from $180 \mathrm{deg}$, or $173.18 \mathrm{deg}$.

It is possible to develop a very simple, numerical index of a cell's directionality. Recall that the magnitudes of sine and cosine terms reflect the displacement of the data centroid from the coordinate system's origin. The displacement along the $\mathrm{x}$ axis is given by the cos term, the displacement along the $y$ axis by the sine term. As in any Euclidean space, the radial distance of the point $(x, y)$ from the origin is given by $\sqrt{x^{2}+y^{2}}$. The larger the distance from the origin, the greater the directional bias in the data. What is meant by directional bias is easiest to see for data which basically consist of two opposite (or nearly opposite) lobes, joined in the neighborhood of the origin. The center of gravity of all the data can be thought of as the balance point between the centers of gravity for the two opposed lobes. In the extreme, when one lobe is vanishingly small, the overall center of gravity may be quite far from the origin and we say that the data show a good deal of directional bias. To be more concrete still, other things being equal, for the Levitt et al. case the value of $\sqrt{r_{\text {sine }}^{2}+r_{\text {cosine } F}^{2}}$ measures the differ- ence in response between the preferred direction [defined by inverse tangent $\left.\left(\mathrm{r}_{\text {sine } F} / \mathrm{r}_{\text {cosine F }}\right)\right]$ and the response to the opposite direction.

A good way to see the meaning of our index of directional bias is to compare the index for each cell with its response profile in Figure 1. For Cell 58-3-1, the most directionally biased of the four, the index is given by the square root of $\left(.0336^{2}+-.2824^{2}\right)$, or .28439. The indices for the other cells are as follows: Cell 57-2-1, .12716; Cell 73-1-1, .06598; and Cell 88-7-1, 00360. A word of warning: the index of directional bias is not a measure of the narrowness of tuning. As we shall see, narrowness is indicated by the presence of higher order harmonics.

\section{INTERPRETATION OF SECOND HARMONIC TERMS}

We can also analyze sine $2 \mathrm{~F}$ and $\cos 2 \mathrm{~F}$ components, keeping in mind that these terms reflect not the eccentricity of the data centroid but the orientation of the data's long axis. In the data of Levitt et al. (Note 2), this corresponds to the preferred orientation of a cell. With both terms nonzero, one-half the inverse tangent $\left(r_{\text {sine }} 2 \mathrm{~F} / \mathrm{r}_{\cos } 2 \mathrm{~F}\right)$ defines the axis of preferred orientation. Again, take Cell 58-3-1 as an example. In Table 1 we find that $\mathrm{r}_{\text {sine } 2 \mathrm{~F}}=-.4392$ and $\mathrm{r}_{\cos 2 \mathrm{~F}}=.3925$. Their ratio is -1.119 ; one-half the inverse tangent of -1.119 is $-24.11 \mathrm{deg}$. The signs of the harmonic components place the axis of orientation in quadrants II and IV. The orientation axis is then $360 \mathrm{deg}$ minus $24.11 \mathrm{deg}$
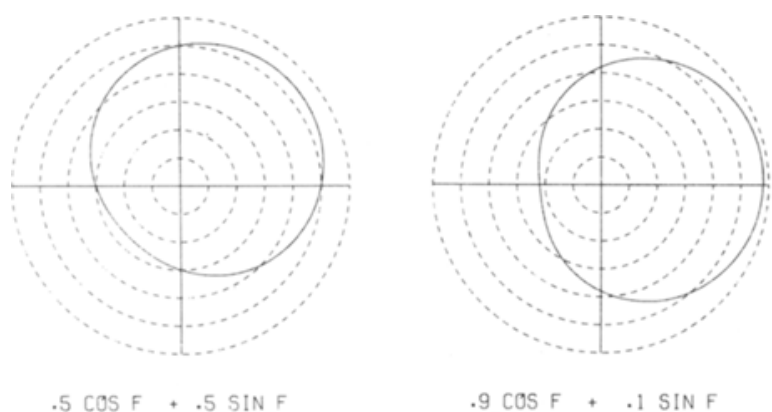

$.5 \cos F+.5 \sin F$ $.9 \cos F+.1 \sin F$
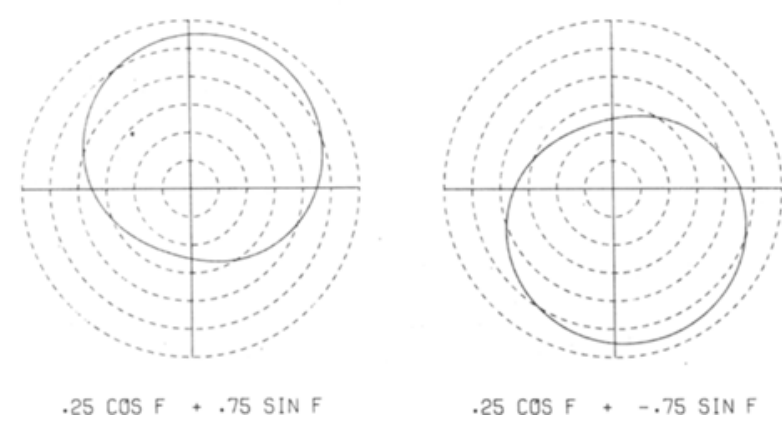

$.25 \cos F+-.75 \operatorname{SIN} F$

Figure 4. Polar plots showing four different combinations of sine $F$ and cosine $F$ components. 
or $335.89 \mathrm{deg}$ (or, equivalently, $180 \mathrm{deg}-24.11 \mathrm{deg}$, or $155.89 \mathrm{deg})$.

Note that for Cell 58-3-1 there is a discrepancy between the direction of the data center's displacement $(173 \mathrm{deg})$ and the axis of preferred orientation $(156 \mathrm{deg})$. This discrepancy, between information based on the fundamental components and information based on the second harmonics, is an index of a form of asymmetry in the data. It indicates that the two lobes of the cell's response do not have precisely the same orientation axis. If the two lobes were perfectly aligned (shared a single axis), the angle of directional bias for the cell would equal the angle of orientation. By comparison, Cell 57-2-1 appears to have nearly the same axis for each lobe: direction bias is at $107 \mathrm{deg}$, axis of orientation is $102 \mathrm{deg}$.

\section{INTERPRETATION OF THE THIRD HARMONIC TERMS}

When we get to the third-order terms of the Fourier series, the interpretation gets more complicated. Although it is an oversimplification, the $3 \mathrm{~F}$ terms reflect greater variance in one-half of the circle than in the other (see Figures $2 \mathrm{c}$ and $3 \mathrm{c}$ ). Actually, the physiological or psychophysical meaning of these higher order terms has to be developed in context. Certain combinations of terms may be needed to represent various complex aspects of the data. For example, the narrowness of tuning-compactness of lobes-depends upon the presence of higher order terms. In the extreme, an infinitely narrowly tuned cell, the lobes would be spikes or delta functions. A narrowly tuned cell would produce a Fourier series heavily weighted on its high-frequency terms. Although such development is beyond the scope of this paper, it should be possible to generate a relatively simple index of narrowness of tuning from a series of high-frequency Fourier terms with appropriate weights. But whatever index one eventually does select might well be validated by comparison against a large series of single cells. An alternate approach, the calculation of circular variance, is given in Mardia (1972).

\section{TEST OF STATISTICAL SIGNIFICANCE}

A word on evaluating the statistical significance of the series' terms. ${ }^{7}$ Recall that $r^{2}$ for any term is the proportion of variance accounted for by that term. As a result, we can use the $\mathrm{r}^{2}$ as the numerator in an F test, with 1 degree of freedom. The simplest defensible denominator for the test is the percent variance not accounted for by the terms analyzed out of the data. To get the total variance accounted for, add up all the $r^{2}$ terms we have computed (in the case of Table 1 or 2 , that would be the sum of the nine $r^{2}$ terms). This sum is then subtracted from 1.0 , giving the total variance not accounted for. The variance unaccounted for pro- vides the denominator sum of square for the $F$ test. The number of degrees of freedom associated with the denominator is the difference between the number of data points [in Levitt et al.'s case (Note 2), 256] and the number of terms analyzed (in Levitt et al.'s case, 9). From here, the $F$ test is standard; divide the denominator SS by its df to get the mean squares for the denominator. Then find the ratio of numerator to denominator and look it up in an $\mathrm{F}$ table.

As one example, consider Cell 58-3-1. The nine terms we analyzed account for .9514 of the variance (Table 1), leaving only .0486 not accounted for. The denominator sum of squares of the $F$ test for any of the nine terms is therefore .0486 and the $\mathrm{df}=256-9=247$. The denominator mean square is therefore $.0486 / 247$, or .0002 . Assuming a $\mathrm{p}$ value of .05 , the critical value of $F$ (with $\mathrm{df}=1 / 247$ ) would be 3.87 . Therefore, any $\mathrm{r}^{2}$ term for the cell would have to exceed $3.87 \cdot(.0002)$, or .00077 , to be statistically significant. Each of the nine terms shown in Table 1 meets this significance criterion. By contrast, only one term (sine $4 \mathrm{~F}$ ) reaches significance for Cell 88-7-1. Although we cannot really justify adopting any one $\alpha$ level rather than any other, we do believe that a liberal level (say, $\alpha=.05$ or .10 ) may be more reasonable than an ultraconservative one. This belief is based upon the fact that in situ a single cortical cell is part of a large array of cells. Even if a cell is only weakly tuned to a particular stimulus property, we cannot rule out the possibility that the cell-taken together with other members of the cortical array-does contribute to the overall neural code for the property in question. Moreover, a more complete characterization, such as that of Table 1, calls attention to the fact that any cortical cell may provide its owner information about more than a single dimension of stimulation. ${ }^{8}$ In general, a characterization of a cell's response properties in neutral terms (like those of a Fourier series) has the advantage of keeping us from thinking of the cell in overly simplistic, anthropocentric terms as "orientation detector" or "movement analyzer."

Before closing, we should briefly consider at least one psychophysical experiment for which our method of analysis is well suited. As part of a larger project, Levinson and Sekuler (1974) measured contrast thresholds for isotropic random dots which moved continuously across a cathode ray tube at $4 \mathrm{deg} / \mathrm{sec}$. Thresholds were measured for each of eight different, equally spaced directions of motion following adaptation to high-contrast dots moving along meridian $315 \mathrm{deg}$. In any session, six thresholds were measured for each test direction using one of two different threshold criteria. With one criterion, the motion threshold, the subject merely had to perceive the motion in the test target; for the higher, pattern threshold, the subject also had to see the spatial structure of the moving target. Since Levinson and Sekuler were interested in the adaptation produced by the high-contrast moving dots, their data 


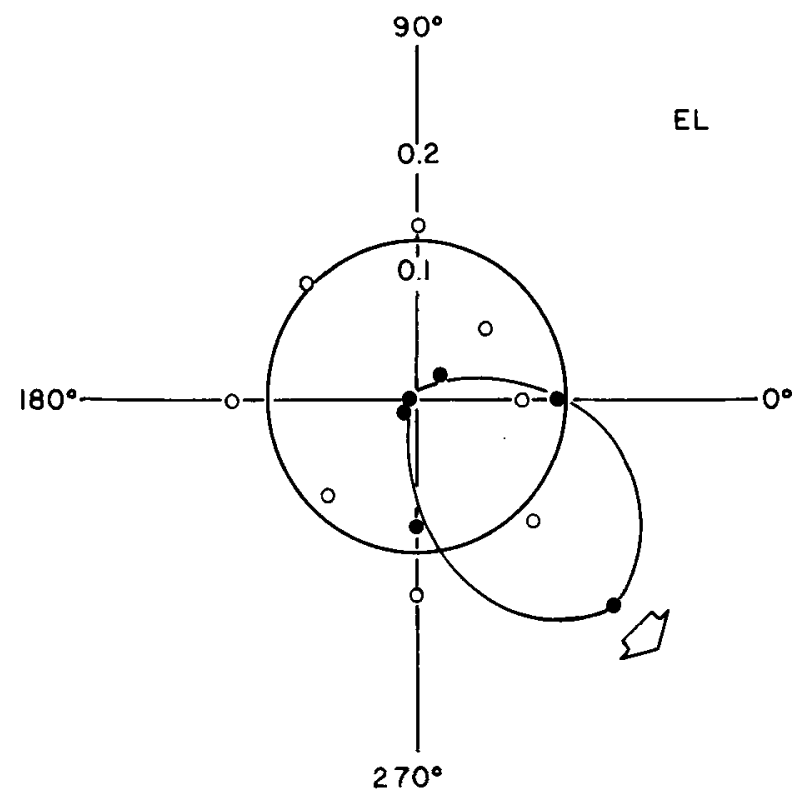

Figure 5. Polar plot of the log threshold elevation produced by adaptation to random dots drifting toward $315 \mathrm{deg}$ (arrow). Test stimuli (also random dots) moved in various directions; the threshold elevation for each test direction represented by the distance from the origin (where threshold elevation is zero). Filled circles are measurements of motion detection, open circles of pattern recognition (from Levinson \& Sekuler, Note 1 ).

were expressed as log (threshold after adaptation/ threshold before adaptation). These values, plotted in Figure 5 for motion and pattern threshold criteria, define the threshold elevations produced by the adaptation.

We subjected pattern and motion threshold data to separate Fourier analyses and will treat primarily the between-condition aspects of the data here. Although we shall not report our entire analysis in detail, where appropriate we shall supplement the discussion of the between-condition Fourier analysis with a consideration of two analyses of variance whose error terms were within-cell variance $(\mathrm{df}=40)$. Consider first the pattern threshold results: The circular term of the series accounted for $95.4 \%$ of all the between-condition variance. An analysis of variance showed the circular term was statistically significant $(p<.01)$, indicating that the moving dots did produce a generalized significant elevation of the pattern threshold. But these elevations of pattern threshold were unrelated to the direction of that movement: None of the individual sine or cosine terms accounted for more than $1 \%$ of the variance. Our index of directional bias confirmed this observation; the index $-\sqrt{\left(r_{\text {sine }}^{2} F+r_{\text {cosine } F}^{2}\right)}$-was virtually zero $(.02)$, indicating that the center of gravity of the data points was essentially at the origin. A quite different picture emerges for the measurements which used a motion criterion. Here the circle term accounts for only $43 \%$ of the between-condition variance, the pair of fundamental terms account for $20 \%$ between them, and the pair of second harmonic terms account for an addi- tional $18 \%$. An analysis of variance showed these terms to be statistically significant (each $p<.01$ ).

The inverse tangent $\left(r_{\text {sine } F} / r_{\text {cosine } F}\right)$ shows that the direction in which the data depart from the origin is 353 deg. Similarly, the orientation axis, defined by one-half the arc tangent $\left(\mathrm{r}_{\text {sine }} 2 \mathrm{~F} / \mathrm{r}\right.$ cosine $\left.2 \mathrm{~F}\right)$, was $318 \mathrm{deg}$. The latter is quite close to the value, $315 \mathrm{deg}$, expected on the basis of the adapting direction used by Levinson and Sekuler (Note 1). The discrepancy between the two axis estimates, 353 and $318 \mathrm{deg}$, suggests that the lobe in quadrant IV defined by the data points is not perfectly symmetrical about the $318 \mathrm{deg}$ meridian. In view of the coarse directional sampling of the independent variable, this difference between the axes is probably not very diagnostic of an actual asymmetry in direction tuning about $315 \mathrm{deg}$. In addition, our index of directional bias is very high: 447 , indicating that, for the motion criterion, adaptation has produced a set of threshold elevations which is highly related to direction. ${ }^{9}$

\section{REFERENCE NOTES}

1. Levinson, E., \& Sekuler, R. Direction-specific adaptation in human vision: Measurements using isotropic random patterns. Presented at the meetings of the Psychonomic Society, Boston, Massachusetts, 1974.

2. Levitt, F. B., Emerson, R. C., \& Brown, J. L. Qualitative examination of effects of strobe rearing on striate receptive fields in cats. Paper presented at the meeting of the Association for Research in Vision and Ophthalmology, Sarasota, Apri] 1977.

\section{REFERENCES}

Adams, A. J., Brown, B., Haegerstrom-Portwoy, G., FLom, M. C., \& Jones, R. T. Evidence for acute effects of alcohol and marijuana on color discrimination. Perception \& Psychophysics, 1976, 20, 119-124.

Appelle, S. Perception and discrimination as a function of stimulus orientation: The "oblique effect" in man and animals. Psychological Bulletin, 1972, 78, 266-278.

Barlow, H. B., Hill, R. M., \& Levick, W. R. Retinal ganglion cells responding selectively to direction and speed of image motion in the rabbit. Journal of Physiology, 1964, 173, 377.407.

Dwass, M. Probability and statistics. New York: W. A. Benjamin, 1970.

Frisen, L., \& Frisen, M. Objective recogntion of abnormal isopters. Acta Ophthalmologica, 1975, 53, 378-392.

Mardia, K. V. Statistics of directional data. New York: Academic Press, 1972.

RoBerts, T. D. M. Neurophysiology of postural mechanisms. New York: Plenum, 1967.

Rodieck, R. W. The vertebrate retina. San Francisco: W. H. Freeman, 1973.

SCHEFFE, H. The analysis of variance. New York: Wiley, 1959.

Sekuler, R. Spatial vision. Annual Review of Psychology, $1974,25,195-232$

Shepard, R. N., \& Cermak, G. W. Perceptual-cognitive explorations of a toroidal set of free-form stimuli. Cognitive Psychology, 1973, 4, 351-377.

ZAR, J. H. Probabilities of Rayleigh's test statistics for circular data. Behavior Research Methods \& Instrumentation, $1974,5,450$. 


\section{NOTES}

1. Polar plots have many other uses in nonvisual areas of sensory physiology. For example, Roberts (1967) used such plots to describe the response of single cells in the ray's utricle to lateral tilt. Other biological uses are given in Mardia (1972).

2. A set of vectors, $a_{1}, \ldots, a_{p}$, is said to span some $n$ dimensional Euclidean space, $S^{n}$, if any vector in $S^{n}$ could be written as a linear combination (or weighted sum) of $a_{1}, \ldots, a_{p}$. To make an analogy with color vision, a set of three primaries, $b, g, r$, spans a three-dimensional space of stimuli if any of the stimuli could be expressed as a weighted sum of the primaries, $k_{1} b+k_{2} g+k_{3} r$. In the present instance, the data for any of Levitt's cells can be thought of as a point in a 256-dimensional space and we seek a set of vectors which, when properly weighted and summed, could describe any possible point in that 256 space. The idea that many different sets of vectors could be used to span a space may be reinforced by reference to color vision. It is well known that there is an infinitely large number of sets of primaries, each of which could span a given color space (Rodieck, 1973, Chapter 23). This simply means that members of any set of primaries could, when properly weighted, describe any stimulus in that color space.

3. $\mathrm{A}$ basis or basis set for the $\mathrm{n}$-dimensional Euclidean space $S^{n}$ is a linearly independent set of vectors, $a_{1}, \ldots, a_{n}$, which span $\mathrm{S}^{n}$. Linear independence implies that none of the vectors could be described by a linear combination of the other vectors. A basis set contains the smallest number of vectors capable of spanning some space, $S^{n}$.

4. For all $\mathrm{m}$, two vectors of sine $\mathrm{mF}$ or cosine $\mathrm{mF}$ are orthogonal so long as the functions are sampled at a uniform interval, $\mathrm{m}$ is integer, and each vector contains an integral number of cycles of the sampled function. The orthogonality of two vectors, $a$ and $b$, is shown by a zero inner product,

$$
\sum_{i=1}^{n} a_{i} b_{i}=0 \text {. }
$$

5. Combining sine and cosine terms at each frequency gives an alternative expression for the Fourier series,

$$
\mathrm{d}_{\mathrm{i}}(\theta)=\mathrm{c}+\sum_{\mathrm{i}=1}^{\mathrm{n}} \mathrm{k}_{\mathrm{i}} \cos \left(\mathrm{i} \frac{\theta}{2 \pi}+\phi_{\mathrm{i}}\right)
$$

where $k_{i}=\sqrt{a_{i}^{2}+b_{i}^{2}}$ and $\phi_{i}=-\tan ^{-1} b_{i} / a_{i}, k_{i}=$ amplitude of the ith component of the Fourier series, and $\phi_{i}=$ phase of the ith component.

6. Readers who may be unfamiliar with statistical trend analysis can think of this procedure as measuring the similarity (i.e., correlation) between the vector of data and each of several specially chosen vectors which contain the values of sine and cosine functions. In our particular case, the measure of similarity happens to run from +1 to -1 . To keep our "similarity" measures within these bounds, we had to normalize data and basis vectors to unit length. As a result, sine and cosine coefficients which we give are proportional to, rather than equal to, the Fourier series coefficients which would have been generated from nonnormalized vectors.

7. The application of inferential statistics to neurophysiological data requires some assumptions which investigators might well be reluctant to make. The most important of these assumptions are (1) that the "noise" is at least approximately Gaussian and (2) that the process being measured does not suffer from severe nonstationarity. As a result, the application of our inferential procedures to neurophysiological data requires greater caution than their application to data from psychophysical studies. An alternate approach to inferential statistics on circular data can be found in Zar (1974) and Mardia (1972).

8. This fact has an important corollary: When a cell's responses are controlled by more than just a single stimulus dimension, it is logically impossible for that cell to provide unambiguous information about visual stimulation along any of those dimensions (see Sekuler, 1974).

9. We would like to call attention to another possible use of polar Fourier series in visual psychophysics. Shepard and Cermak (1973) describe the potential utility of Fourier synthesized outline representations of natural objects (such as a cat).
(Received for publication July 29,1977 ; revision accepted November $22,1977$. ) 